\title{
An elementary proof of the Ambartzumian-Pleijel identity
}

\author{
BY A. J. CABO \\ CWI, P.O. Box 4079, 1009 AB Amsterdam, The Netherlands
}

(Received 30 April 1991; revised 20 February 1992)

\section{Introduction}

In [5], Pleijel proved an identity relating the area $A$ of a convex plane domain and the length $L$ of its boundary (of class $C^{1}$ ). In particular, it contains the isoperimetric inequality $L^{2}-4 \pi A \geqslant 0$.

Ambartzumian gave two proofs of a generalized version of the Pleijel-identity for convex polygons. The first proof (in [1]) consisted of direct computations. In his book [2] however, he shows that the identity is an easy consequence of the solution to the Buffon-Sylvester problem.

Pohl proved an analogous formula for closed convex plane curves with smooth boundary, applying Stokes' theorem to a suitable manifold with boundary (see [6]).

The aim of this note is to show that Stokes' theorem may also be used to prove Ambartzumian's Pleijel-type identity for convex polygons directly. It turns out that the use of differential forms leads to considerable simplifications. The interesting question whether this method may be used to derive a Pleijel-type identity for more general convex domains, remains unanswered.

\section{Ambartzumian's Pleijel-type identity for convex polygons}

Throughout this section, let $C$ denote a (bounded) closed convex polygon in the plane. The main idea of the proof is to compute the integral of a differential form over two of the sides of $C$. Then by a limiting procedure the result follows immediately.

To be able to perform the integration, we have to give an orientation to the sides.

Let $a$ and $b$ be two non-intersecting sides of $C$ that do not share any of their endpoints $A_{1}, A_{2}$ and $B_{1}, B_{2}$ respectively. The set $a \times b$ is a two-dimensional submanifold of $\mathbb{R}^{4}$, which can be parametrized in the following way. Let $u$ and $v$ be the vectors $A_{2}-A_{1}$ and $B_{2}-B_{1}$ respectively. Then $x \in a$ and $y \in b$ have the representation

$$
x=A_{1}+\theta_{1} u, \quad y=B_{1}+\theta_{2} v,
$$

for some numbers $\theta_{1}, \theta_{2} \in[0,1]$.

If $d l_{1}$ (resp. $d l_{2}$ ) is the element of length in $a$ (resp. $b$ ), directed from $A_{1}$ to $A_{2}$ (resp. $B_{1}$ to $B_{2}$ ), then the 2 -form $d l_{1} \wedge d l_{2}$ has the representation

$$
d l_{1} \wedge d l_{2}=|a| \cdot|b| d \theta_{1} \wedge d \theta_{2}
$$

where $d \theta_{1} \wedge d \theta_{2}$ is the canonical 2 -form on $\mathbb{R}^{2}$ and $|x|$ denotes the length of the side $x$.

Using this parametrization, we can consider $a \times b$ as an oriented manifold with boundary. Define the mapping $\phi:[0,1]^{2} \rightarrow a \times b$ by

$$
\phi\left(\theta_{1}, \theta_{2}\right)=\left(A_{1}+\theta_{1} u, B_{1}+\theta_{2} v\right) .
$$


Then we have

$$
a \times b=\phi\left([0,1]^{2}\right)
$$

and the oriented boundary of $a \times b$ is identified by this mapping with the boundary in $\mathbb{R}^{2}$ of the unit square with the usual counter-clockwise orientation. From this identification, it is seen that $a \times\left\{B_{1}\right\}$ and $\left\{A_{2}\right\} \times b$ have the same orientation as $a, b$ respectively, and that $a \times\left\{B_{2}\right\}$ and $\left\{A_{1}\right\} \times b$ have the opposite orientation.

We shall need the following lemma in the proof.

LEMMA. Let $a$ and $b$ be as described above and let $(x, y)$ be a point on $a \times b$. Let $d l_{1}, d l_{2}$ denote the element of length on $a$ and $b$ respectively and let $\chi$ denote the length of the segment joining $x$ and $y$, that is directed from $x$ to $y$. Furthermore, let $\alpha_{1}$ and $\alpha_{2}$ be the angles, lying to the right of $\chi$, formed by $\chi$ and the sides $a$ and $b$ respectively.

Then we have, for fixed $y$

$$
d \alpha_{1}=\frac{\sin \alpha_{2}}{\chi} d l_{2}
$$

and for fixed $x$

$$
d \alpha_{2}=-\frac{\sin \alpha_{1}}{\chi} d l_{1}
$$

Proof. First fix $l_{1}$. Let $h_{x}$ be the length of the perpendicular from $x$ onto $b$. Then

$$
\frac{h_{x}}{-l_{2}}=\tan \left(\pi-\alpha_{2}\right)=-\tan \alpha_{2}
$$

hence

$$
\alpha_{2}=\arctan \frac{h_{x}}{l_{2}}
$$

Consequently

$$
\frac{d \alpha_{2}}{d l_{2}}=-\frac{h_{x}}{l_{2}^{2}+h_{x}^{2}}=-\frac{h_{x}}{\chi^{2}}=-\frac{\sin \alpha_{2}}{\chi}
$$

Since clearly $\alpha_{1}+\alpha_{2}$ is constant, the first assertion follows.

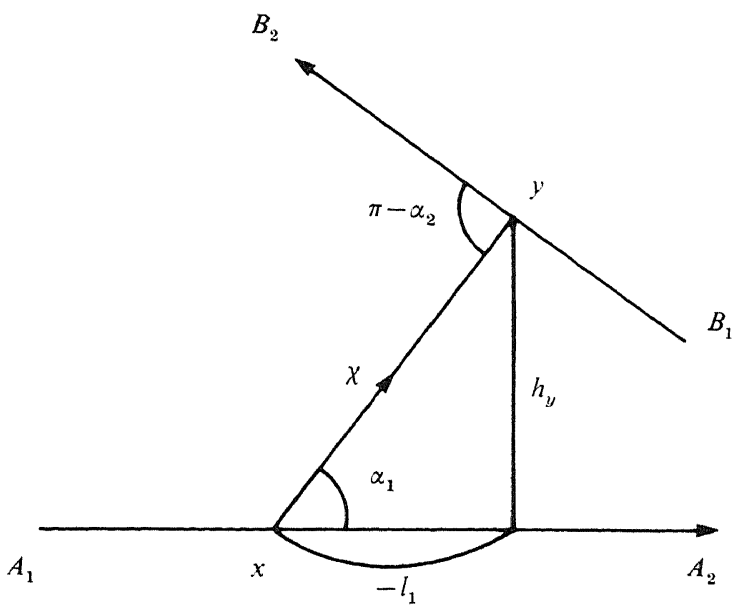

Fig. 1 
Next, fix $l_{2}$ and let $h_{y}$ be defined similarly to $h_{x}$. Then

$$
\frac{d \alpha_{2}}{d l_{1}}=-\frac{d \alpha_{1}}{d l_{1}}=-\frac{d}{d l_{1}} \arctan \left(-\frac{h_{y}}{l_{1}}\right)=-\frac{\sin \alpha_{1}}{\chi}
$$

This proves the lemma.

Observe that if $l_{2}$ increases, for $l_{1}$ fixed, then the angle $\alpha_{1}$ increases. On the other hand, if $l_{1}$ increases, for $l_{2}$ fixed, then the angle $\alpha_{2}$ decreases. As a consequence, we see that the signs of (1) and (2) are correct.

We are now ready to prove the Pleijel-type identity.

Theorem (Ambartzumian-Pleijel). Let $\mathrm{C}$ be a convex polygon with $n$ sides $a_{i}$ of length $\left|a_{i}\right|$. Suppose that $C$ is oriented as described above. Let $f: \mathbb{R} \rightarrow \mathbb{R}$ be a $C^{1}$-function. Then

$$
\int_{[C]} f(\chi) d g=\int_{[C]} f^{\prime}(\chi) \chi \cot \alpha_{1} \cot \alpha_{2} d g+\sum_{i=1}^{n} \int_{0}^{\left|a_{i}\right|} f(x) d x,
$$

where $d g$ denotes the element of an invariant measure on the set $G$ of non-oriented lines in the plane and $[C]:=\{g \in G: g \cap C \neq \varnothing\}$.

Proof. First consider two sides $a$ and $b$ with endpoints $A_{1}, A_{2}$ and $B_{1}, B_{2}$ respectively. Suppose that $a$ and $b$ are non-intersecting but not parallel and that they do not share an endpoint.

Consider the orientation-preserving differential form $d l_{1} \wedge d l_{2}$, where $d l_{1}$ (resp. $d l_{2}$ ) is the element of length along $a$ (resp. $b$ ), as defined above. Define the 1 -form $\omega$ on $a \times b$ by

Then

$$
\omega(x, y)=\cos \alpha_{1} d l_{1}+\cos \alpha_{2} d l_{2} .
$$

$$
d \omega=-\sin \alpha_{1} d \alpha_{1} \wedge d l_{1}-\sin \alpha_{2} d \alpha_{2} \wedge d l_{2} .
$$

By the Lemma, (3) may be written as

$$
d \omega=-\frac{\sin \alpha_{2}}{\chi} \sin \alpha_{1} d l_{2} \wedge d l_{1}+\frac{\sin \alpha_{1}}{\chi} \sin \alpha_{2} d l_{1} \wedge d l_{2}
$$

whence, by the anti-commutativity of the exterior product

$$
d \omega=2 \frac{\sin \alpha_{1} \sin \alpha_{2}}{\chi} d l_{1} \wedge d l_{2}
$$

Define $\omega_{1}:=f(\chi) \omega$. Then we may apply Stokes' theorem (see e.g. [4]) to the 1 -form $\omega_{1}$ on $a \times b$, since the latter is an oriented 2 -manifold with boundary. This yields

$$
\int_{\hat{c}(a \times b)} \omega_{1}=\int_{a \times b} d \omega_{1}=\int_{a \times b} f^{\prime}(\chi) d \chi \wedge \omega+\int_{a \times b} f(\chi) d \omega .
$$

Observe that

$$
\frac{-d \chi}{d l_{2}}=\cos \left(\pi-\alpha_{2}\right)=-\cos \alpha_{2}
$$

hence

$$
d \chi=\cos \alpha_{2} d l_{2} \text {. }
$$

Analogously, we have

$$
d \chi=-\cos \alpha_{1} d l_{1} \text {. }
$$

Consequently

$$
d \chi \wedge \omega=d \chi \wedge \cos \alpha_{1} d l_{1}+d \chi \wedge \cos \alpha_{2} d l_{2}=-2 \cos \alpha_{1} \cos \alpha_{2} d l_{1} \wedge d l_{2} .
$$


By (4),

$$
\int_{a \times b} f(\chi) d \omega=2 \int_{a \times b} \frac{\sin \alpha_{1} \sin \alpha_{2}}{\chi} d l_{1} \wedge d l_{2}
$$

Hence (5) may be written as

$$
\int_{a \times b} f(x) \frac{\sin \alpha_{1} \sin \alpha_{2}}{\chi} d l_{1} \wedge d l_{2}=\int_{a \times b} \cos \alpha_{1} \cos \alpha_{2} f^{\prime}(\chi) d l_{1} \wedge d l_{2}+\frac{1}{2} \int_{\partial(a \times b)} f(\chi) \omega .
$$

At the beginning of the section, we showed that the boundary $\partial(a \times b)$ of $a \times b$ is

Consequently

$$
\bigcup_{i=1,2}\left(a \times\left\{B_{i}\right\}\right) \cup \bigcup_{i=1,2}\left(\left\{A_{i}\right\} \times b\right) .
$$

$$
\left.\begin{array}{rl}
\int_{\partial(a \times b)} f(x) \omega= & \int_{\left\{A_{1} \backslash \times b\right.} f(\chi) \omega+\int_{\left\{A_{2}\right\} \times b} f(\chi) \omega+\int_{a \times\left\{B_{1}\right\}} f(\chi) \omega+\int_{a \times\left\{B_{2}\right\}} f(\chi) \omega \\
= & -\int_{\left\{A_{1}\right\} \times b} f(\chi) \cos \alpha_{2} d l_{2}+\int_{\left\{A_{2}\right\} \times b} f(\chi) \cos \alpha_{2} d l_{2} \\
& +\int_{a \times\left\{B_{1}\right\}} f(\chi) \cos \alpha_{1} d l_{1}-\int_{a \times\left\{B_{2}\right\}} f(\chi) \cos \alpha_{1} d l_{1},
\end{array}\right\}
$$

where one has to take the orientation into consideration. Equation (7) corresponds to equation (21) in [1], in a version for directed lines.

Next, we let the endpoint $B_{1}$ of $b$ tend to the endpoint $A_{2}$ of $a$, i.e. the distance between $B_{1}$ and $A_{2}$ tends to zero. Then in the limit, where $A_{2}=B_{1}$, we get

$$
\int_{\partial(a \times b)} f(x) \omega=\int_{0}^{|a|} f(x) d x+\int_{0}^{|b|} f(x) d x-\int_{\left.a \times i B_{2}\right\}} f(x) \cos \alpha_{1} d l_{1}-\int_{\left\{A_{1}\right\} \times b} f(x) \cos \alpha_{2} d l_{2} .
$$

Summation of (6) over all sides of $C$, using (8) as well as the lemma, completes the proof of the theorem. Observe that indeed terms of the form $\int_{0}^{\left|a_{i}\right|} f(x) d x$ appear twice in the sum. Furthermore, there is cancellation of terms of the form

as desired.

$$
\int_{\left\{A_{i} \backslash \times a_{j}\right.} f(\chi) \cos \alpha_{2} d l_{2} \text { and } \int_{a_{i} \times\left\{A_{j}\right\}} f(\chi) \cos \alpha_{1} d l_{1}
$$

This note is a version of one of the sections of the author's master's thesis [3]. The author would like to thank P. Groeneboom again, under whose supervision she had the pleasure of writing it.

\section{REFERENCES}

[1] R. V. Ambar'tzumian. Convex polygons and random tessellations. In Stochastic Geometry: A Tribute to the memory of Rollo Davidson (Wiley, 1974), pp. 176-191.

[2] R. V. Ambar'tzumian. Combinatorial Integral Geometry (Wiley, 1982).

[3] A. J. CABO. (hordlength distributions and characterization problems for convex plane polygons. Master's thesis, University of Amsterdam (1989).

[4] V. Gulllemin and A. Pollack. Differential Topology (Prentice-Hall, 1974).

[5] A. PleiJel. Zwei kurze Beweise der isoperimetrischen Ungleichung. Arch. Math. (Basel) 7 (1956), 317-319.

[6] W. F. PoHL. The probability of linking of random closed curves. In Geometry Symposium Utrecht, Lecture Notes in Math. vol. 894 (Springer-Verlag, 1980), pp. 113-126. 